Research Paper

\title{
Expression of ARIDIB Is Associated With Poor Outcomes and Predicts the Benefit from Adjuvant Chemotherapy in Bladder Urothelial Carcinoma
}

\author{
Beihe Wang1,4*, Huyang Xie ${ }^{1,4 *}$, Chunguang Ma1,4, Guiming Zhang5, Hualei Gan², 4, Qifeng Wang2, 4 ,

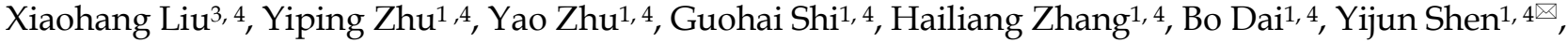 \\ Dingwei $\mathrm{Ye}^{1,4 \bowtie}$ \\ 1. Department of Urology, Fudan University Shanghai Cancer Center, Shanghai, 200032, China; \\ 2. Department of Pathology, Fudan University Shanghai Cancer Center, Shanghai, 200032, China; \\ 3. Department of Radiology, Fudan University Shanghai Cancer Center, Shanghai, 200032, China; \\ 4. Department of Oncology, Shanghai Medical College, Fudan University, Shanghai, 200032, China; \\ 5. Department of Urology, The Affiliated Hospital of Qingdao University, Shandong, 266003, China. \\ * These authors contributed equally to this work. \\ $\square$ Corresponding authors: Dr. Yijun Shen (yijunshen79@163.com) and Dr. Dingwei Ye (dwyeli@163.com), Department of Urology, Fudan University Shanghai \\ Cancer Center, 270 Dong' an Rd. (M), Shanghai 200032, China. Tel: +86-21-64175590; fax: +86-21-64434556 \\ (c) Ivyspring International Publisher. This is an open access article distributed under the terms of the Creative Commons Attribution (CC BY-NC) license \\ (https://creativecommons.org/licenses/by-nc/4.0/). See http://ivyspring.com/terms for full terms and conditions.
}

Received: 2017.01.08; Accepted: 2017.06.06; Published: 2017.09.27

\begin{abstract}
Background ARIDIB, which exists as a mutually exclusive isoform with ARIDIA in the SWI/SNF chromatin remodeling complex, has been recently identified as a major mutant gene in a wide variety of cancers. The present study aimed to determine the association between ARIDIB expression and outcomes, as well as the benefit from adjuvant chemotherapy in patients with bladder cancer.

Methods Tissue microarrays of 143 consecutively recruited patients with bladder cancer from our center were created. Immunohistochemistry was performed to assess the expression of ARIDIB and its association with outcomes. Clinicopathological factors were also evaluated.

Results ARIDIB expression was significantly associated with tumor size $(P=0.015)$, T stage $(P=0.027)$, lymph node status $(P=0.030)$, TNM stage $(P=0.040)$, overall survival $(P<0.001)$, and progression-free survival $(P=0.043)$. Furthermore, high expression of ARIDIB was an independent indicator of poor OS $(P=0.022)$. The prognostic model containing ARIDIB showed a better predictive accuracy than the bench models. Most importantly, the benefit of adjuvant chemotherapy observed in patients with low ARIDIB expression was superior to that observed in patients with high ARIDIB expression.

Conclusions Our study suggests that ARIDIB can serve as a prognostic biomarker of bladder urothelial carcinoma. Additionally, ARIDIB might be a predictive marker for selecting patients for adjuvant chemotherapy in the high-risk subgroup.
\end{abstract}

Key words: bladder cancer, prognosis, biomarker, adjuvant chemotherapy.

\section{Introduction}

Bladder cancer is the fourth most common cancer with an incidence of approximately $7 \%$, and the eighth most common cause of mortality in men (approximately 4\%) [1]. In 2015, 80,500 new bladder cancer cases are expected, with 32,900 estimated deaths for both sexes in China [2]. Despite considerable progress in bladder cancer treatment, the prognosis of patients with locally advanced bladder cancer remains poor because of the heterogeneity of disease characteristics [3]. This situation has resulted in a need for accurate prognostic assessment after radical surgery that is essential for treatment decision making, counseling of patients, and most importantly, for defining the indication of adjuvant chemotherapy 
[4].

Among these prognostic models, the American Joint Committee on Cancer (AJCC) TNM staging system is the most widely used prognostic model to predict outcome in patients treated with radical cystectomy [5]. These staging systems provide useful estimates of outcome. However, a major flaw of these systems is the difficulty in incorporating novel clinical information, such as molecular markers or more complex bioinformatics. Furthermore, the current staging systems are less accurate than some prediction models incorporating several clinical data in the era of personalized medicine [6].

Switch/sucrose non-fermentable (SWI/SNF) complex is a family of chromatin remodeling factors, which play crucial roles in cell differentiation, proliferation, DNA repair, and many other cellular processes [7]. The SWI/SNF complex is composed of two major sub-classes, BRG1/BRM-associated factor and polybromo-associated BRG1/BRM-associated factor complexes. The well-conserved core subunits contained in BRG1/BRM-associated factor and polybromo-associated BRG1/BRM-associated factor, such as BRG1 or BRM, are the main catalytic subunits for ATPase activity. In the BRG1/BRM-associated factor complex, AT-rich interactive domaincontaining protein 1A (ARID1A) and 1B (ARID1B) exist as mutually exclusive isoforms [8-10]. ARID1A contributes to cell-cycle arrest [11], and interacts with p53 and BRG1/BRM [12], and with topoisomerase Iia [13], suggesting that ARID1A is an important tumor suppressor. Mutations in the ARID1B gene, which shares approximately $60 \%$ similarities in amino acid sequence with ARID1A, play an important role in causing Coffin-Siris syndrome. This syndrome shows developmental defects and intellectual disability [14-16]. More importantly, recent studies have identified mutant alleles of ARID1B by whole-genome or whole-exome surveys in human cancers, such as colorectal, pancreatic, and breast cancer, and neuroblastoma.

This study aimed to determine the association between ARID1B expression and patients' outcome by using bladder urothelial carcinoma tissue microarrays (TMAs) and study the effect of ARID1B in bladder cancer cells.

\section{Materials and Methods}

\section{Patients and clinical database}

A consecutive cohort of 143 patients with bladder cancer treated with radical cystectomy were retrospectively recruited between March 2008 and December 2012 in Fudan University Shanghai Cancer Center. The patients' clinical characteristics, laboratory data, and treatment plan were obtained from in-patient records. The follow-up information was collected by a trained study nurse. The study protocol was approved by the institutional review board of Fudan University Shanghai Cancer Center and the study was carried out according to the approved guidelines. Each patient was well informed with the details of this study and informed consent was obtained.

Patients were selected based on the criteria of 1) an age of 18 years or older, 2) confirmed histopathological diagnosis, and 3) reviewed TNM classification according to the 2010 AJCC. Other inclusion criteria included possession of paraffin-embedded tissue blocks available for immunohistochemical staining and complete outcome data. Of the 143 patients, 7 were excluded for the absence of paraffin-embedded tissue blocks and 5 were excluded for incomplete follow-up data. Overall survival (OS) was calculated from the date of surgery to the date of death or last contact. Progression-free survival (PFS) was defined as the time from surgery to the date of progression. A professional radiologist assessed responses and progression, which were defined by standard criteria. The median follow-up for the entire cohort was 64.5 months $(95 \%$ CI: 56.4-72.6).

\section{Immunohistochemistry}

Tissue microarrays were constructed as described previously. Primary anti-ARID1B antibody ( $3 \mu \mathrm{g} / \mathrm{ml}$, ab57461; Abcam, Cambridge, UK) was used for immunohistochemical staining. Two independent pathologists who were blinded to the clinicopathological data and outcome of each patient evaluated the staining intensity of the specimen. A semi-quantitative immunoreactivity scoring system was used for this evaluation as reported elsewhere. X-tile software (version 3.6.1, Yale University, New Haven, CT) was applied to select the optimum cutoff score for the staining intensity to separate patients into high and low ARID1B expression groups.

\section{Western-blot assay}

Cells and tissues were lysed in RIPA buffer (150 $\mathrm{mM} \mathrm{NaCl}, 50 \mathrm{mM}$ Tris- $\mathrm{HCl}$ (pH 7.2), 0.5\% NP-40, 1\% Triton $\mathrm{X}-100,1 \%$ sodium deoxycholate) containing a protease inhibitor cocktail (Sigma-Aldrich Corporation, MO, USA). Cell and tissue lysates were separated on sodium dodecyl sulfate (SDS)-polyacrylamide gels and transferred to an Immun-Blot ${ }^{\circledR}$ polyvinylidene fluoride membrane (Bio-Rad Laboratories, Inc. CA, USA). The membranes were blocked with $5 \%$ non-fat milk in TBST and subsequently probed with primary ARID1B 
antibodies (1:1000, ab57461; Abcam, Cambridge, UK) overnight at $4^{\circ} \mathrm{C}$. Mouse anti-b-actin monoclonal antibody (1:3000; cat. no. 60008; Proteintech Group, Inc., IL, USA) was used as an internal control. Primary antibodies were detected by incubating the membranes with a horseradish peroxidaseconjugated secondary antibody (1:3000; ab6728; Abcam, Cambridge, UK) for $1 \mathrm{~h}$ at room temperature. The blots were subsequently developed using an enhanced chemiluminescence detection kit (Thermo Fisher Scientific, Inc. IL, USA) and exposure to film.

\section{Cell Culture and silencing of ARIDIB}

T24 bladder cancer cells were cultured in McCoy's 5a medium supplemented with $10 \%$ fetal bovine serum. According to the mRNA sequence of ARID1B in GenBank (Gene ID: 57492), two siRNAs (si1\# and si2\#) were designed. The specific siRNAs and the negative control siRNA (siNC) were synthesized by Guangzhou RiboBio company. The siRNA sequences are shown in Table S1. The cells were plated on a six-well plate, and then transfected with human ARID1B siRNA according to the manufacturer's transfection protocol.

\section{Cell proliferation assay}

Cell proliferation was determined with the WST-8 assay using Cell Counting kit-8 (Dojindo, Kumamoto, Japan), in which 1,000 cells/well were placed in a 96-well plate. After 24, 48, 72, 96 and 120h, $10 \mu 1$ of Cell Counting kit-8 solution [2-(2-methoxy-4-nitrophenyl)-3-(4-nitrophenyl)-5-(2,4 -disulfophenyl)-2H-tetrazolium, monosodium salt] was added to each well and incubated for $2 \mathrm{~h}$. Cell viability was determined by reading the optical density in each well at $450 \mathrm{~nm}$.

\section{Cell migration and invasion assay}

Cell migration and invasion were measured using transwell chambers (Corning, NY, USA) containing 24-well inserts with $8 \mu \mathrm{m}$ pores in the presence or absence of Matrigel (BD Biosciences, NY, USA) according to the manufacturer's protocol. At $48 \mathrm{~h}$ after transfection, T24 cells were incubated for an additional $30 \mathrm{~h}$ for migration or $48 \mathrm{~h}$ for invasion. Then, the cells in the upper chamber were removed, and the remaining cells were fixed in $4 \%$ paraformaldehyde and stained with crystal violet solution. Cells were quantified in four randomly selected fields for each membrane.

\section{Statistical analysis}

Categorical data, presented as frequencies and percentages, were analyzed using Fisher's exact chi-square test. Continuous data, presented as mean and range, were analyzed by the Student's t-test.
Associations between endpoints and potential prognostic factors were assessed by using the log-rank test in univariate analysis. The Cox proportional hazards model was undertaken subsequently in multivariate analysis to assess the independent effect of the variables. Hazard ratios (HRs) and the 95\% CIs of covariates were calculated. The accuracy of the prognostic factors was evaluated by Harrell's concordance index (C-index). All statistical analyses were performed using $\mathrm{R}$ software (https://www.r-project.org). The level of statistical significance was set at $P<0.05$, and all $P$ values are two-sided.

\section{Results}

\section{ARIDIB expression levels and their association with patients' clinical characteristics}

To investigate whether ARID1B expression is related to the outcome of bladder urothelial carcinoma, immunohistochemistry was applied to evaluate its expression. ARID1B-positive staining was predominantly located in the nucleus and presented as a dot-shaped stain with a variable amount of staining (Figure 1). We used western blotting to validate the immunohistochemistry results (Figure S1) and to study ARID1B expression in tumor tissue and normal tissue. ARID1B expression was higher in tumor tissues than in paired adjacent normal tissues (Figure S2). We also found that patients with T4 diseases had a higher rate of high ARID1B expression than did patients with other stages of disease. (Table S2). Then, the entire cohort was separated into the high ARID1B expression group $(\mathrm{n}=34)$ and low ARID1B expression group $(n=97)$ according to the cutoff value that was derived from the immunoreactivity scoring score by X-tile. The correlations between ARID1B expression and clinicopathological features are shown in Table 1. ARID1B expression was significantly associated with tumor size $(P=0.015)$, $\mathrm{T}$ stage $(P=0.027)$, lymph node status $(P=0.030)$, and TNM stage $(P=0.040)$.

\section{High expression of ARIDIB is associated with OS and PFS, and is an independent indicator of poor OS in patients with bladder urothelial carcinoma}

Kaplan-Meier curves showed that patients with high ARID1B expression had a significantly poorer OS $(P<0.001$, Figure 2$)$ and PFS $(P=0.043)$ than those with low ARID1B expression. Univariate and multivariate COX analyses of all of the clinicopathological variables and ARID1B expression were performed to investigate whether the prognostic value of ARID1B expression was independent of 
well-known prognostic indicators (Table 2 and Table S3).

Tumor grade $(\mathrm{HR}=2.929,95 \% \mathrm{CI}: 1.052-8.149$, $P=0.040)$, high ARID1B expression $(\mathrm{HR}=2.813,95 \% \mathrm{CI}$ : 1.594-4.964, $P<0.001)$, adjuvant chemotherapy (HR=1.865, 95\% CI: 1.042-3.336, $P=0.036$ ), T stage (per increase in stage: $\mathrm{HR}=2.197,95 \% \mathrm{CI}: 1.558-3.099$, $P<0.001)$, lymph node status $(\mathrm{HR}=2.995,95 \% \mathrm{CI}$ : 1.691-5.305, $P<0.001$ ), and TNM stage (per increase in stage: $\mathrm{HR}=1.926,95 \% \mathrm{CI}: 1.466-2.564, P<0.001)$ were risk factors in univariate analysis (Table 2). Therefore, these factors might predict poor OS in patients with bladder urothelial carcinoma treated with radical cystectomy.

The multivariate Cox proportional hazards model was performed involving all of the potential factors that were identified by univariate analysis, except for TNM stage, because it is derived from $\mathrm{T}$ stage and lymph node status. Using backward elimination, besides tumor grade, $\mathrm{T}$ stage, and lymph node status, ARID1B expression (HR=1.986, 95\%CI:1.105-3.571, $P=0.022$ ) was identified as an independent prognostic factor for OS (Table 2).

To investigate whether this finding was dependent on $\mathrm{T}$ stage, we performed a subgroup analysis by $\mathrm{T}$ stage. ARID1B expression was strongly associated with $\mathrm{OS}$ in patients with $\mathrm{T} 1+\mathrm{T} 2$ stage $(\mathrm{HR}=5.181,95 \%$ CI:2.094-12.822, $P<0.001$; Figure S3 A, B and C).
Table 1. The association between ARIDIB expression and patients' clinical characteristics

\begin{tabular}{|c|c|c|c|c|c|}
\hline \multirow[t]{2}{*}{ Characteristic } & \multicolumn{2}{|l|}{ Patient } & \multicolumn{3}{|c|}{ ARID1B expression } \\
\hline & NO. & $\%$ & Low & High & $P$ \\
\hline Age, years & & & & & 0.706 \\
\hline Mean \pm SD & $60.6 \pm 10$. & & $60.3 \pm 10.3$ & $61.1 \pm 9.4$ & \\
\hline Median(range) & $61(33-83)$ & & $61(33-83)$ & $61.5(35-8$ & \\
\hline Gender & & & & & 0.686 \\
\hline Male & 117 & 89.3 & 86 & 31 & \\
\hline Female & 14 & 10.7 & 11 & 3 & \\
\hline Tumor size, $\mathrm{cm}$ & & & & & 0.015 \\
\hline Mean \pm SD & $3.8 \pm 1.9$ & & $3.6 \pm 1.9$ & $4.5 \pm 1.7$ & \\
\hline Range & $0.7-9.5$ & & $0.7-9.5$ & $1.7-9.0$ & \\
\hline Tumor grade & & & & & 0.151 \\
\hline Low & 22 & 16.8 & 19 & 3 & \\
\hline High & 109 & 83.2 & 78 & 31 & \\
\hline Adjuvant chemotherapy & & & & & 0.267 \\
\hline Positive & 33 & 25.2 & 22 & 11 & \\
\hline Negative & 98 & 74.8 & 75 & 23 & \\
\hline Disease pattern & & & & & \\
\hline Primary & 89 & 67.9 & 66 & 23 & 0.967 \\
\hline Relapse & 42 & 32.1 & 31 & 11 & \\
\hline Number of original tumo & & & & & 0.410 \\
\hline Solitary & 81 & 61.8 & 62 & 19 & \\
\hline Multiple & 50 & 38.2 & 35 & 15 & \\
\hline T stage & & & & & 0.027 \\
\hline 1 & 21 & 16.0 & 18 & 3 & \\
\hline 2 & 57 & 43.5 & 43 & 14 & \\
\hline 3 & 38 & 29.0 & 29 & 9 & \\
\hline 4 & 15 & 11.5 & 7 & 8 & \\
\hline Lymph nodes status & & & & & 0.030 \\
\hline Negative & 102 & 77.9 & 78 & 21 & \\
\hline Positive & 29 & 22.1 & 19 & 13 & \\
\hline TNM stage & & & & & 0.040 \\
\hline 1 & 21 & 16.0 & 17 & 4 & \\
\hline 2 & 50 & 38.2 & 40 & 10 & \\
\hline 3 & 22 & 16.8 & 17 & 5 & \\
\hline 4 & 38 & 29.0 & 23 & 15 & \\
\hline
\end{tabular}

\section{Magnification}

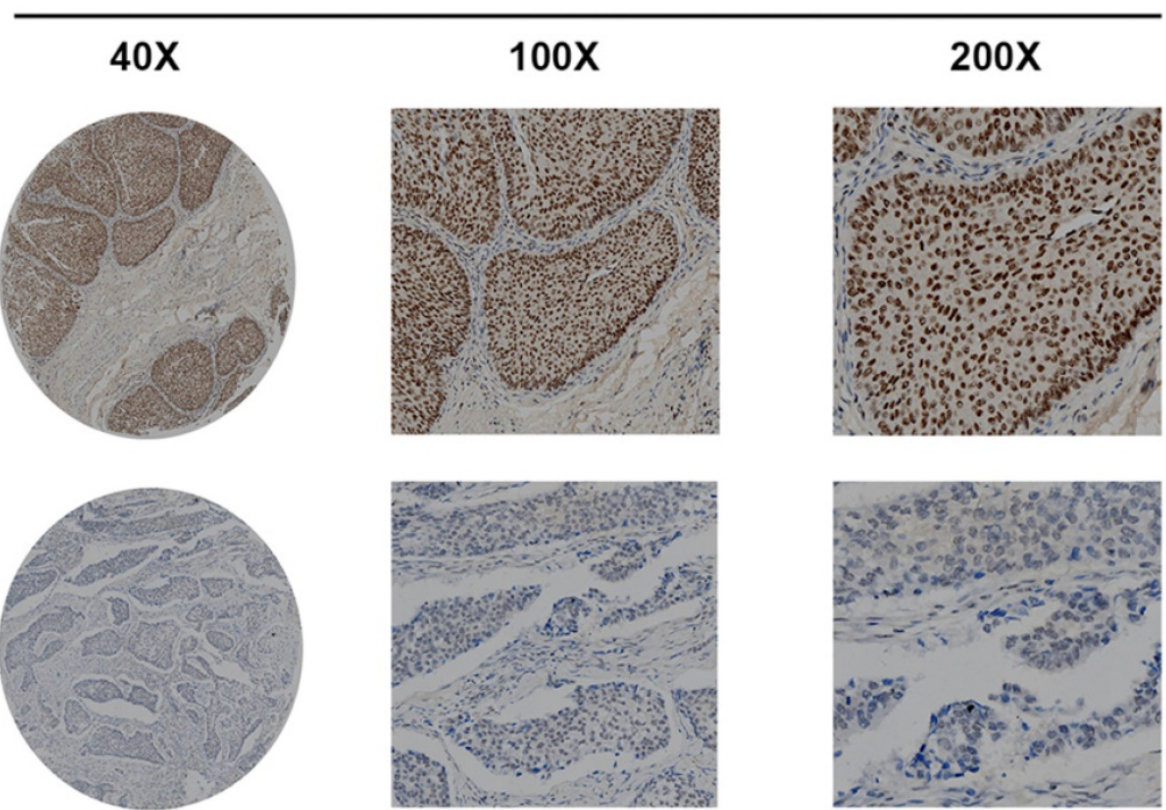

Figure 1. ARIDIB expression in bladder urothelial carcinoma tissue. Representative ARIDIB immunohistochemistry images show high expression levels (upper panel) and low expression levels (lower panel). 
A

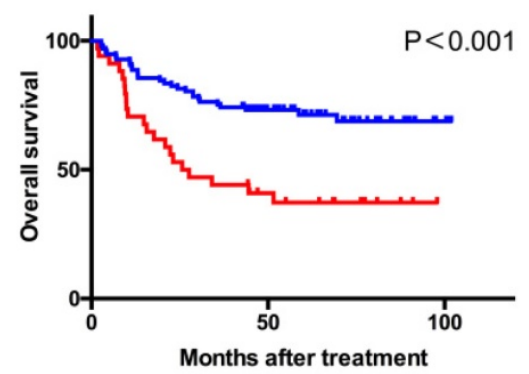

B

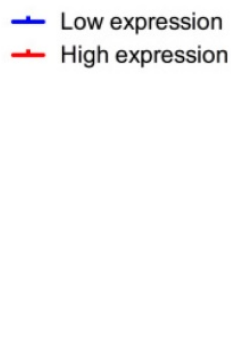

B

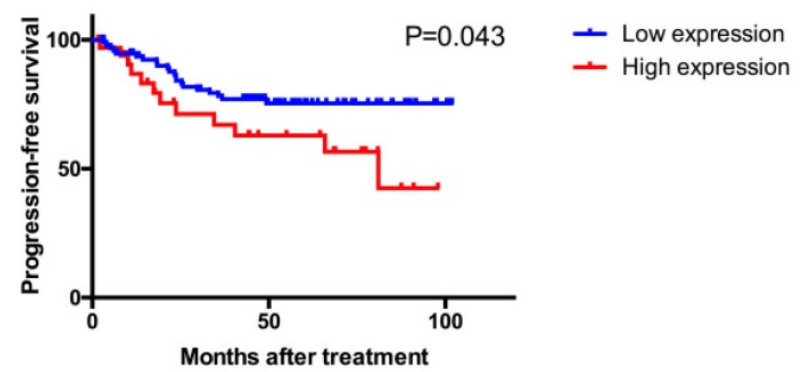

Figure 2. Overall survival (OS) and progression-free survival (PFS) analysis of patients with bladder urothelial carcinoma based on ARIDIB expression. Kaplan-Meier analysis of OS (A) and PFS (B).

Table 2. Univariate and multivariate Cox regression analysis of overall survival.

\begin{tabular}{|c|c|c|c|c|c|c|c|c|c|}
\hline \multirow[t]{2}{*}{ Characteristics } & \multicolumn{3}{|c|}{ Univariate } & \multicolumn{3}{|c|}{ Multivariate (Full model) } & \multicolumn{3}{|c|}{ Multivariate (Reduced model) } \\
\hline & $\overline{\mathrm{HR}}$ & $95 \% \mathrm{CI}$ & $P$ & $\mathrm{HR}$ & $95 \% \mathrm{CI}$ & $P$ & HR & $95 \% \mathrm{CI}$ & $P$ \\
\hline Age & 1.022 & $0.990-1.055$ & 0.185 & & & & & & \\
\hline \multicolumn{10}{|l|}{ Gender } \\
\hline Male & Ref. & & & & & & & & \\
\hline Female & 0.887 & $0.351-2.239$ & 0.799 & & & & & & \\
\hline Tumor size & 1.027 & $0.890-1.184$ & 0.716 & & & & & & \\
\hline \multicolumn{10}{|l|}{ Tumor grade } \\
\hline Low & Ref. & & & Ref. & & & Ref. & & \\
\hline High & 2.929 & $1.052-8.149$ & 0.040 & 3.868 & $1.354-11.055$ & 0.012 & 3.817 & $1.340-10.874$ & 0.012 \\
\hline \multicolumn{10}{|l|}{ ARID1B expression } \\
\hline Low & Ref. & & & Ref. & & & Ref, & & \\
\hline High & 2.813 & $1.594-4.964$ & $<0.001$ & 1.970 & $1.093-3.548$ & 0.024 & 1.986 & $1.105-3.571$ & 0.022 \\
\hline \multicolumn{10}{|l|}{ Adjuvant chemotherapy } \\
\hline Negative & Ref. & & & Ref. & & & - & - & - \\
\hline Positive & 1.865 & $1.042-3.336$ & 0.036 & 0.898 & $0.467-1.729$ & 0.749 & - & - & - \\
\hline \multicolumn{10}{|l|}{ T stage } \\
\hline Per increase in stage & 2.197 & $1.558-3.099$ & $<0.001$ & 1.896 & $1.265-2.842$ & 0.002 & 1.862 & $1.257-2.758$ & 0.002 \\
\hline \multicolumn{10}{|l|}{ Lymph nodes status } \\
\hline Negative & Ref. & & & Ref. & & & Ref. & & \\
\hline Positive & 2.995 & $1.691-5.305$ & $<0.001$ & 2.110 & $1.076-4.137$ & 0.030 & 2.045 & $1.072-3.903$ & 0.030 \\
\hline \multicolumn{10}{|l|}{ TNM stage } \\
\hline Per increase in stage & 1.926 & $1.466-2.564$ & $<0.001$ & & & & & & \\
\hline
\end{tabular}

\section{Extension of prognostic models with ARID IB expression for patients with bladder urothelial carcinoma}

To further assess the prognostic power of ARID1B expression, we constructed a prognostic model including all of the variables that remained in the reduced model in Table 2 . We then compared the predictive accuracy of this model with TNM stage by C-index analysis. The C-index was 0.692 (95\% CI: $0.550-0.835$ ) when assessed with the TNM stage model and was improved to 0.724 (95\% CI: 0.585-0.863) when ARID1B expression was added (Table 3). The C-index for the new model that we constructed, including ARID1B expression, tumor grade, $\mathrm{T}$ stage, and lymph node status, was 0.754 (95\% CI: $0.627-0.881$ ). This showed the best predictive accuracy among all of the models.

\section{ARIDIB expression and the benefit from adjuvant chemotherapy}

To evaluate which subgroup of patients might benefit from adjuvant chemotherapy, we pooled the patients who received adjuvant chemotherapy together with high-risk patients $(\mathrm{pT} 3+$ disease or a positive lymph node) who did not. Among all patients with bladder urothelial carcinoma, treatment with adjuvant chemotherapy was not associated with a higher rate of 5-year OS $(P=0.407)$. However, treatment with adjuvant chemotherapy was strongly associated with a higher rate of 5-year OS in the low ARID1B expression group $(P=0.030)$, but not in the high ARID1B expression group $(P=0.398)$. A test for the interaction between the biomarker and treatment showed that the benefit observed in the low ARID1B group was superior to that observed in the high ARID1B expression group ( $P$ for interaction $=0.027$; Figure 3). 


\section{ARIDI B knockdown suppresses bladder cancer cell proliferation, migration and invasion}

Cancer proliferation, migration and invasion are frequently associated with cancer heterogeneity and are important factors for cancer management. We studied ARID1B expression levels in four available bladder cancer cell lines (Figure S4) and selected T24 cells (which are widely used in bladder cancer research) to study the function of ARID1B. Transfection of the T24 cells with siRNA against ARID1B resulted in an apparent knockdown of the gene (Figure 4A). ARID1B inhibition caused significant decreases in cell proliferation, migration ability and invasiveness (Figure 4B-D).

Table 3. Comparison of the accuracy of the prognostic models for overall survival

\begin{tabular}{lll}
\hline Model & C-index & $95 \% \mathrm{CI}$ \\
\hline ARID1B & 0.613 & $0.481-0.745$ \\
TNM stage & 0.692 & $0.550-0.835$ \\
ARID1B+TNM stage & 0.724 & $0.585-0.863$ \\
ARID1B+T stage +lymph nodes status +tumor grade & 0.754 & $0.627-0.881$ \\
\hline
\end{tabular}
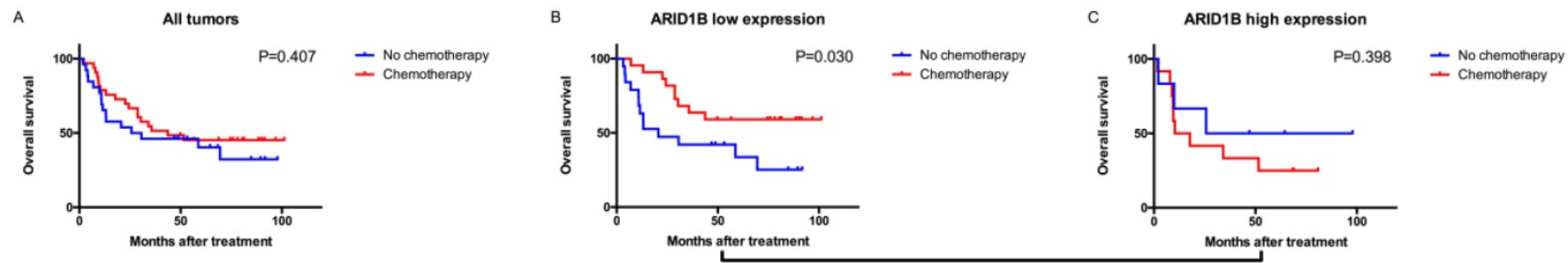

$\mathrm{P}=0.027$ for interaction

Figure 3. Relationship between ARIDIB expression and benefit from adjuvant chemotherapy. Kaplan-Meier analysis of OS in all high-risk patients (A), low ARIDIB expression patients (B), and high ARIDIB expression patients (C).
A

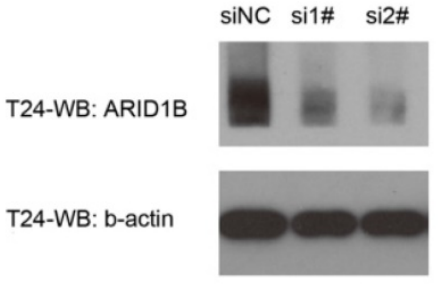

B

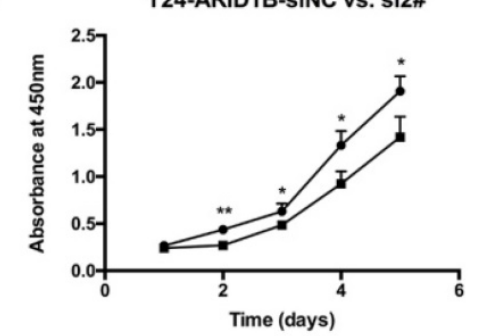

T24-ARID1B-siNC vs. si2\#

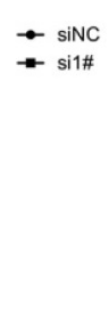

$\rightarrow$ sinc

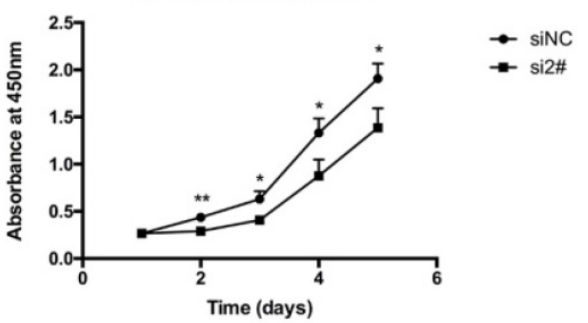

C

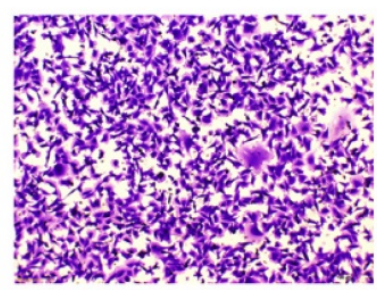

T24-ARID1B-siNC
T24-ARID1B-si1\#

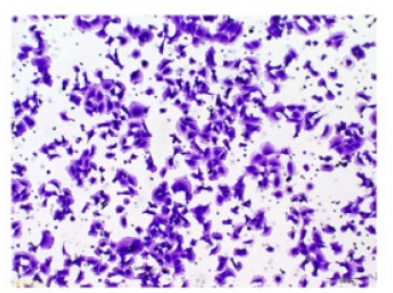

T24-ARID1B-si2\#

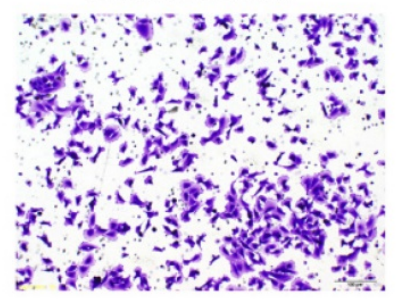

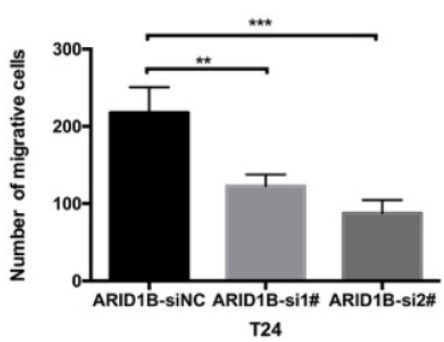

T24

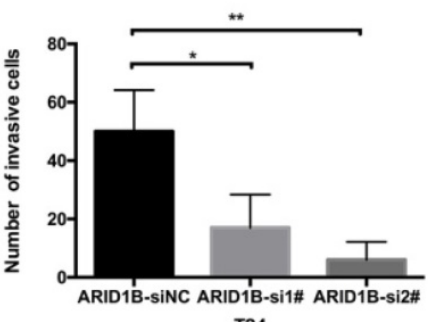

T24

Figure 4. Effect of ARIDIB inhibition on cell proliferation, migration and invasion. The knockdown efficiency of ARIDIB in T24 bladder cancer cells treated with control siRNA (siNC), ARIDIB siRNA I\# (sil\#) and siRNA 2\# (si2\#) (A). Inhibition of ARIDIB in T24 cells significantly decreased cell proliferation (B), cell migration $(C)$ and cell invasion $(D) . * P<0.05, * * P<0.01$ and $* * * P<0.001$ 


\section{Discussion}

To the best of our knowledge, we showed for the first time that high ARID1B expression was positively associated with tumor size, $\mathrm{T}$ stage, lymph node status, TNM stage, and an increased risk of death, as well as disease progression, in these patients. Furthermore, we extended the prognostic model by including ARID1B expression and showed a better predictive accuracy compared with the AJCC TNM staging system. Most importantly, we demonstrated that patients with low ARID1B expression might benefit from adjuvant chemotherapy. We also showed that the knockdown of ARID1B caused significantly decreased cell proliferation, migration and invasion ability in T24 cells, which correlated with the possible role of ARID1B as an oncogene in bladder cancer. Taken together, our findings suggest that ARID1B can serve as a prognostic biomarker of bladder urothelial carcinoma. Additionally, ARID1B might help with selecting adjuvant chemotherapy after radical cystectomy.

The SWI/SNF chromatin remodeling complex, including ARID1A and ARID1B, plays essential roles in repairing DNA damage. Chromatin remodeling propagates DNA damage signals within cells and facilitates access of DNA repair proteins to DNA damage in chromatin [17]. Adjuvant chemotherapy used in this study included standard cisplatin-based regimens: methotrexate, vinblastine, doxorubicin and cisplatin (MVAC) with growth factor support; gemcitabine and cisplatin; or cisplatin, methotrexate and vinblastine (CMV). Actual dosages were determined by patients' tolerance. Watanabe et al. used live-cell analysis and gene suppression experiments to show that suppression of either ARID1A or ARID1B leads to increased sensitivity to cisplatin [18]. This finding indicates that cancer cells lacking in expression of ARID1A and ARID1B are deficient in DNA repair and potentially vulnerable to DNA damage. This may be an explanation for our finding that patients with low ARID1B expression benefited more from adjuvant chemotherapy. Over the past 3 decades, there have been many clinical trials that aimed to evaluate the efficacy of adjuvant chemotherapy in locally advanced bladder cancer [19-22]. However, because of different flaws and selection of regimens, they were either underpowered or yielded conflict results. As a result, the role of adjuvant chemotherapy remains controversial and all international guidelines are restrictive with regard to its recommendation. The European Association of Urology guidelines state that "neither randomized trials nor a meta-analysis has provided sufficient data to support the routine use of chemotherapy." [23]
They supported a category $2 \mathrm{~B}$ recommendation in the guidelines of the National Comprehensive Cancer Network. Recently, Galsky et al. compared the efficacy of adjuvant chemotherapy versus observation post-cystectomy using propensity score analysis [24]. They found an association between adjuvant chemotherapy and improved survival. Consistent with their study, our results could help better select patients who could potentially benefit from adjuvant chemotherapy.

Recent studies have suggested that SWI/SNF factors, especially ARID1A and ARID1B, might encode products involved in carcinogenesis by identifying the inactivating mutant alleles in different cancers and primary cancer cells [25]. Helming et al. suggested that ARID1B confers a specific vulnerability in ARID1A-mutant cancers, and the loss of ARID1B in ARID1A-deficient backgrounds destabilizes SWI/SNF and impairs proliferation [26]. Additionally, ARID1A and ARID1B may play opposing roles in tumorigenesis. ARID1A significantly contributes to tumor suppression activities characterized by anti-proliferation function. Conversely, ARID1B plays a wide-ranging role in promoting proliferation and maintaining stemness in mouse embryonic stem cells [27, 28]. In contrast, Khrsheed et al. demonstrated that overexpression of ARID1B severely compromised the ability of pancreatic cancer cell lines to form colonies [29]. As a result, the possible role that ARID1B plays in tumorigenesis still remains unclear. Recently, Shao et al. showed that highly expressed ARID1B was observed in aggressive breast cancer subtypes and was associated with a poor disease-free survival rate in patients with breast cancer [30]. Consistent with this previous study, we showed that high ARID1B expression predicted poor OS in patients with bladder urothelial carcinoma. This finding indicates that ARID1B may promote progression in bladder cancer.

The major limitations of this study were its retrospective design, it was performed in a single center, and there was a relatively small number of patients. However, the proposed prognostic model appeared to improve the predictive accuracy. Additionally, we demonstrated that ARID1B plays an important role in adjuvant chemotherapy and the inhibition of ARID1B caused significant suppression in cell proliferation, migration and invasion in bladder cancer cells. Our results still need further experiments and validation in prospective cohorts or clinical trials.

\section{Supplementary Material}

Supplementary figures and tables. http://www.jcancer.org/v08p3490s1.pdf 


\section{Acknowledgements}

This study was funded by grants from the National Natural Science Foundation of China (81272837, 81472377, 81302216, 81672512 and 81572531). The study sponsor had no role in the study design, collection, analysis, and interpretation of data. We would like to thank all of the patients who participated in this study.

\section{Competing Interests}

The authors have declared that no competing interest exists.

\section{References}

1. Siegel RL, Miller KD, Jemal A. Cancer statistics, 2015. CA Cancer J Clin. 2015; 65: 5-29.

2. Chen W, Zheng R, Baade PD, Zhang S, Zeng H, Bray F, et al. Cancer statistics in China, 2015. CA Cancer J Clin. 2016; 66: 115-32.

3. Kaufman DS, Shipley WU, Feldman AS. Bladder cancer. Lancet. 2009; 374: 239-49.

4. Leow JJ, Martin-Doyle W, Rajagopal PS, Patel CG, Anderson EM, Rothman AT, et al. Adjuvant chemotherapy for invasive bladder cancer: a 2013 updated systematic review and meta-analysis of randomized trials. Eur Urol. 2014; 66: $42-54$

5. Edge SB, Compton CC. The American Joint Committee on Cancer: the 7th edition of the AJCC cancer staging manual and the future of TNM. Ann Surg Oncol. 2010; 17: 1471-4.

6. Kluth LA, Black PC, Bochner BH, Catto J, Lerner SP, Stenzl A, et al. Prognostic and Prediction Tools in Bladder Cancer: A Comprehensive Review of the Literature. Eur Urol. 2015; 68: 238-53.

7. Phelan ML, Sif S, Narlikar GJ, Kingston RE. Reconstitution of a core chromatin remodeling complex from SWI/SNF subunits. Mol Cell. 1999; 3: 247-53.

8. Wang W, Xue Y, Zhou S, Kuo A, Cairns BR, Crabtree GR. Diversity and specialization of mammalian SWI/SNF complexes. Genes Dev. 1996; 10: 2117-30.

9. Inoue H, Furukawa T, Giannakopoulos S, Zhou S, King DS, Tanese N. Largest subunits of the human SWI/SNF chromatin-remodeling complex promote transcriptional activation by steroid hormone receptors. J Biol Chem. 2002; 277: 41674-85

10. Wang X, Nagl NG, Wilsker D, Van Scoy M, Pacchione S, Yaciuk P, et al. Two related ARID family proteins are alternative subunits of human SWI/SNF complexes. Biochem J. 2004; 383: 319-25.

11. Nagl NG, Jr., Patsialou A, Haines DS, Dallas PB, Beck GR, Jr., Moran E. The p270 (ARID1A/SMARCF1) subunit of mammalian SWI/SNF-related complexes is essential for normal cell cycle arrest. Cancer Res. 2005; 65: 9236-44

12. Guan B, Wang TL, Shih Ie M. ARID1A, a factor that promotes formation of SWI/SNF-mediated chromatin remodeling, is a tumor suppressor in gynecologic cancers. Cancer Res. 2011; 71: 6718-27.

13. Dykhuizen EC, Hargreaves DC, Miller EL, Cui K, Korshunov A, Kool M, et al. BAF complexes facilitate decatenation of DNA by topoisomerase IIalpha. Nature. 2013; 497: 624-7.

14. Halgren C, Kjaergaard S, Bak M, Hansen C, El-Schich Z, Anderson CM, et al. Corpus callosum abnormalities, intellectual disability, speech impairment, and autism in patients with haploinsufficiency of ARID1B. Clin Genet. 2012; 82: $248-55$

15. Hoyer J, Ekici AB, Endele S, Popp B, Zweier C, Wiesener A, et al. Haploinsufficiency of ARID1B, a member of the SWI/SNF-a chromatin-remodeling complex, is a frequent cause of intellectual disability. Am J Hum Genet. 2012; 90: 565-72.

16. Santen GW, Aten E, Sun Y, Almomani R, Gilissen C, Nielsen M, et al. Mutations in SWI/SNF chromatin remodeling complex gene ARID1B cause Coffin-Siris syndrome. Nature genetics. 2012; 44: 379-80.

17. Seeber A, Hauer M, Gasser SM. Nucleosome remodelers in double-strand break repair. Curr Opin Genet Dev. 2013; 23: 174-84.

18. Watanabe R, Ui A, Kanno S, Ogiwara H, Nagase T, Kohno T, et al. SWI/SNF factors required for cellular resistance to DNA damage include ARID1A and ARID1B and show interdependent protein stability. Cancer Res. 2014; 74: $2465-75$

19. Freiha F, Reese J, Torti FM. A randomized trial of radical cystectomy versus radical cystectomy plus cisplatin, vinblastine and methotrexate chemotherapy for muscle invasive bladder cancer. J Urol. 1996; 155: 495-9; discussion 9-500.

20. Stockle M, Meyenburg W, Wellek S, Voges G, Gertenbach U, Thuroff JW, et al. Advanced bladder cancer (stages pT3b, pT4a, pN1 and pN2): improved survival after radical cystectomy and 3 adjuvant cycles of chemotherapy.
Results of a controlled prospective study. J Urol. 1992; 148: 302-6; discussion 6-7

21. Sabichi AL, Lerner SP, Atkinson EN, Grossman HB, Caraway NP, Dinney CP, et al. Phase III prevention trial of fenretinide in patients with resected non-muscle-invasive bladder cancer. Clin Cancer Res. 2008; 14: 224-9.

22. Sternberg CN, Skoneczna I, Kerst JM, Albers P, Fossa SD, Agerbaek M, et al. Immediate versus deferred chemotherapy after radical cystectomy in patients with pT3-pT4 or N+ M0 urothelial carcinoma of the bladder (EORTC 30994): an intergroup, open-label, randomised phase 3 trial. Lancet Oncol. 2015; 16: 76-86.

23. Witjes JA, Comperat E, Cowan NC, De Santis M, Gakis G, Lebret T, et al. EAU guidelines on muscle-invasive and metastatic bladder cancer: summary of the 2013 guidelines. Eur Urol. 2014; 65: 778-92.

24. Galsky MD, Stensland KD, Moshier E, Sfakianos JP, McBride RB, Tsao CK, et al. Effectiveness of Adjuvant Chemotherapy for Locally Advanced Bladder Cancer. J Clin Oncol. 2016; 34: 825-32.

25. Wu JN, Roberts CW. ARID1A mutations in cancer: another epigenetic tumor suppressor? Cancer discovery. 2013; 3: 35-43.

26. Helming KC, Wang $X$, Wilson BG, Kaplan-Meier analysis of overall survival (OS, panel A) and progression-free survival (PFS, panel B) of patients with bladder urothelial carcinoma based on ARID1B expression. Vazquez $\mathrm{F}$, Haswell JR, Manchester HE, et al. ARID1B is a specific vulnerability in ARID1A-mutant cancers. Nature medicine. 2014; 20: 251-4.

27. Nagl NG, Jr., Wang X, Patsialou A, Van Scoy M, Moran E. Distinct mammalian SWI/SNF chromatin remodeling complexes with opposing roles in cell-cycle control. EMBO J. 2007; 26: 752-63.

28. Yan Z, Wang Z, Sharova L, Sharov AA, Ling C, Piao Y, et al. BAF250B-associated SWI/SNF chromatin-remodeling complex is required to maintain undifferentiated mouse embryonic stem cells. Stem Cells. 2008; 26: 1155-65.

29. Khursheed M, Kolla JN, Kotapalli V, Gupta N, Gowrishankar S, Uppin SG, et al. ARID1B, a member of the human SWI/SNF chromatin remodeling complex, exhibits tumour-suppressor activities in pancreatic cancer cell lines. Br J Cancer. 2013; 108: 2056-62.

30. Shao F, Guo T, Chua PJ, Tang L, Thike AA, Tan PH, et al. Clinicopathological significance of ARID1B in breast invasive ductal carcinoma. Histopathology. 2015; 67: 709-18 